\title{
The role of lung inflation and sodium transport in airway liquid clearance during lung aeration in newborn rabbits
}

\author{
Melissa L. Siew' ${ }^{1}$, Megan J. Wallace', Beth J. Allison ${ }^{1,8}$, Marcus J. Kitchen², Arjan B. te Pas ${ }^{3}$, M. Sirajul Islam², Robert A. Lewis ${ }^{4,5}$, \\ Andreas Fouras ${ }^{6}$, Naoto Yagi ${ }^{7}$, Kentaro Uesugi ${ }^{7}$ and Stuart B. Hooper ${ }^{1}$
}

BACKGROUND: Recent phase-contrast X-ray imaging studies suggest that inspiration primarily drives lung aeration and airway liquid clearance at birth, which questions the role of adrenaline-induced activation of epithelial sodium channels (ENaCs). We hypothesized that pressures generated by inspiration have a greater role in airway liquid clearance than do ENaCs after birth.

METHODS: Rabbit pups (30 d of gestation) were delivered and sedated, and $0.1 \mathrm{ml}$ of saline (S) or amiloride (Am; an ENaC inhibitor) was instilled into the lungs before mechanical ventilation. Two other groups (30 d of gestation) were treated similarly but were also given adrenaline (S/Ad and Am/Ad) before mechanical ventilation.

RESULTS: Amiloride and adrenaline did not affect functional residual capacity $(F R C)$ recruitment $(P>0.05)$. Amiloride increased the rate of FRC loss between inflations (Am: $-5.2 \pm$ $0.6 \mathrm{ml} / \mathrm{kg} / \mathrm{s}$ ), whereas adrenaline reduced the rate of FRC loss (S/Ad: $-1.9 \pm 0.3 \mathrm{ml} / \mathrm{kg} / \mathrm{s}$ ) as compared with saline-treated controls (S: $-3.5 \pm-0.6 \mathrm{ml} / \mathrm{kg} / \mathrm{s} ; P<0.05)$.

CONCLUSION: These data indicate that inspiration is a major determinant of airway liquid clearance and FRC development during positive pressure ventilation. Although $\mathrm{ENaC}$ inhibition and adrenaline administration had no detectable effect on FRC development, ENaC may help to prevent liquid from re-entering the airways during expiration.

$\mathbf{A}^{\mathrm{t}}$ $\mathrm{t}$ birth, airway liquid clearance and lung aeration initiate cardiopulmonary changes, and together they underpin the transition to newborn life. Airway liquid clearance is accomplished by a variety of mechanisms (1-3), depending on the mode and timing of delivery. However, cell culture $(4,5)$, animal $(6-8)$, and human $(9,10)$ studies imply that adrenaline-induced activation of pulmonary epithelial sodium channels (ENaCs) is the primary mechanism. This predicts that increased $\mathrm{Na}^{+}$ reabsorption from the lung lumen reverses the osmotic gradient across the pulmonary epithelium, favoring liquid movement from the airways into the interstitial tissue (11-13). However, no studies have assessed the independent effects of $\mathrm{ENaC}$ inhibition vs. other factors such as increased transpulmonary hydrostatic pressures generated by inspiration. These transpulmonary pressures refer to the pressure difference across the pulmonary epithelium between the distal airways and the surrounding interstitial tissue.

Phase-contrast (PC)X-ray imaging exploits the refractive index difference between air and water to generate high-resolution images of the aerating lung after birth. As X-rays pass through the aerated lung, they are refracted at each air-liquid interface, which generates a characteristic "speckle" pattern from which lung gas volumes can be determined (14-16). Together with plethysmography, PC X-ray imaging has demonstrated the dependence of lung aeration on inspiratory activity after birth $(17,18)$. These data have questioned the role of $\mathrm{Na}^{+}$reabsorption and suggest that inspiration may be the primary force promoting airway liquid clearance after birth. However, because PC X-ray imaging visualizes only air/liquid boundaries, it is possible that air and liquid initially coexist in aerated airways and that airway liquid clearance is slower than indicated by imaging (18).

In this study, we investigated the independent roles of $\mathrm{ENaC}$ activation and transpulmonary pressures on lung aeration in mechanically ventilated near-term rabbit pups. Simultaneous PC X-ray imaging and plethysmography were used to measure the development and maintenance of lung gas volumes in pups given amiloride (an $\mathrm{ENaC}$ antagonist) in the presence or absence of adrenaline (which activates ENaCs) (Study A). In a separate experiment, we investigated the relationship between lung aeration and airway liquid clearance to confirm that significant volumes of liquid do not coexist with air in aerated distal airways (Study B).

\section{RESULTS}

\section{Study A}

Animal data. PC X-ray images and plethysmograph recordings were collected from 20 pups from a total of four pregnant rabbits. Pups administered saline $(S ; n=5)$ and amiloride (Am; $n=4)$ into the lungs weighed $43.6 \pm 2.8 \mathrm{~g}$, whereas pups administered saline into the lungs and adrenaline into the

\footnotetext{
'The Ritchie Centre, Monash Institute of Medical Research, Monash University, Clayton, Australia; ${ }^{2}$ School of Physics, Monash University, Clayton, Australia; ${ }^{3}$ Department of Pediatrics, Leiden University Medical Centre, Leiden, The Netherlands; ${ }^{4}$ Divison of Biomedical Engineering, University of Saskatchewan, Saskatoon, Saskatchewan, Canada; ${ }^{5}$ Department of Medical Imaging and Radiation Science, Monash University, Clayton, Australia; ${ }^{6}$ Division of Biological Engineering, Monash University, Clayton, Australia; ${ }^{7}$ SPring-8/Japanese Synchrotron Radiation Institute, Sayo, Japan; ${ }^{8}$ Current address: Department of Physiology, Development and Neuroscience, University of Cambridge, Cambridge, UK. Correspondence: Melissa L. Siew (melissa.siew@monash.edu)
} 

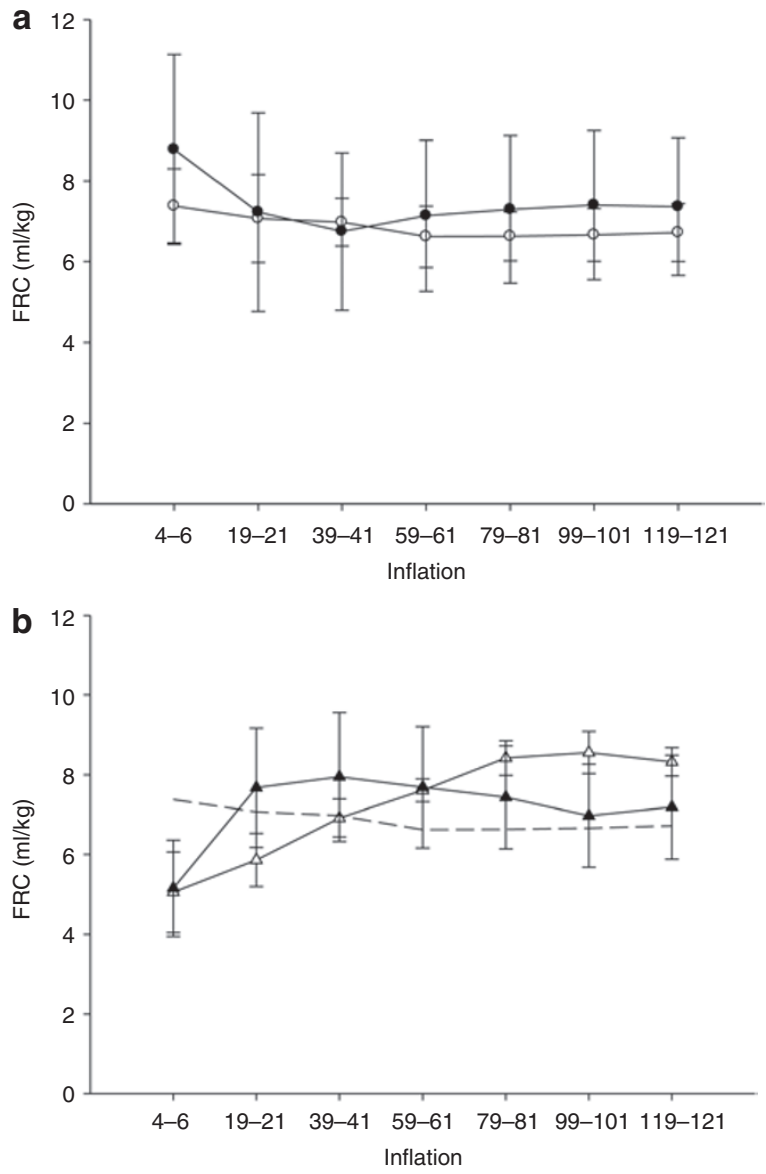

Figure 1. FRC development. FRC measured in (a) pups treated with saline (S, open circles) or amiloride (Am, filled circles) or (b) pups administered adrenaline and then treated with saline ( $\mathrm{S} / \mathrm{Ad}$, open triangles) or amiloride (Am/Ad, filled triangles). The dashed line represents FRC from S-treated pups in a. FRC, functional residual capacity.

umbilical vein (S/Ad; $n=7$ ) and pups administered amiloride into the lungs and adrenaline into the umbilical vein $(\mathrm{Am} / \mathrm{Ad}$; $n=4)$ weighed $36.6 \pm 1.2 \mathrm{~g}(P<0.05)$. If the plethysmograph leaked or adrenaline administration was unsuccessful, the pup was excluded from this analysis $(n=4)$.

Functional residual capacity. Amiloride had no effect on the development of functional residual capacity (FRC) as measured by plethysmography and PC X-ray images. At inflations $4-6$, FRC was $7.4 \pm 0.9 \mathrm{ml} / \mathrm{kg}$ in $\mathrm{S}$ pups and $8.8 \pm 2.4 \mathrm{ml} / \mathrm{kg}(P$ $>0.50$ ) in Am pups (Figure 1a). FRC did not increase in either group after this time $(P>0.05)$. Adrenaline administration in the presence or absence of amiloride did not affect the development or maintenance of FRC. FRC at inflations 4-6 was 5.2 $\pm 1.2 \mathrm{ml} / \mathrm{kg}$ in $\mathrm{Am} / \mathrm{Ad}$ and $5.1 \pm 1.0 \mathrm{ml} / \mathrm{kg}$ in S/Ad pups $(P>$ 0.05 ) (Figure 1b). By 119-121 inflations, FRC remained similar between groups $(P>0.05)$.

FRC loss. FRC loss between inflations, starting from the inflection point of each breath to the end of expiration (Figure 2a), was greater in Am than in S pups. From inflations 20-40, FRC loss in S pups decreased from $-4.7 \pm 0.6$ to $-3.5 \pm 0.6 \mathrm{ml} / \mathrm{kg} / \mathrm{s}$
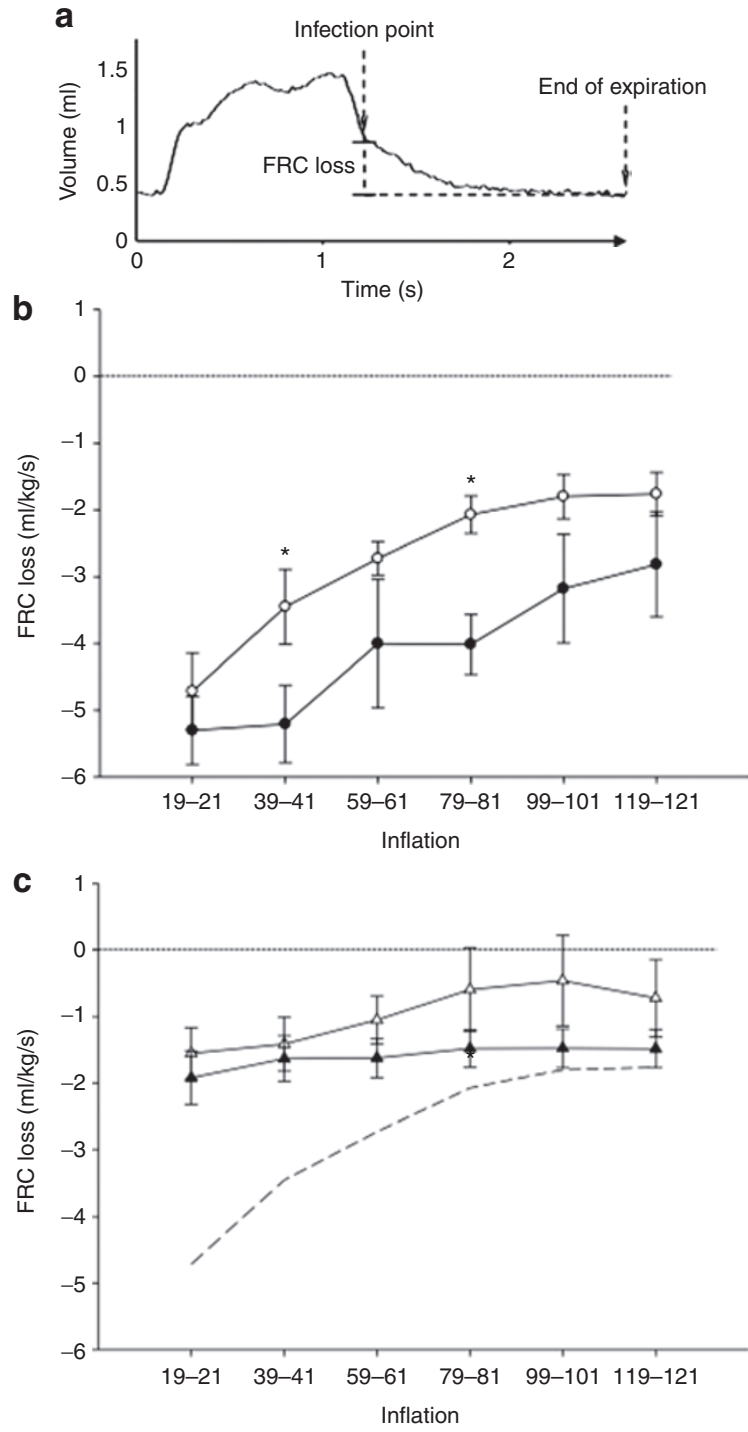

Figure 2. FRC loss between inflations. (a) Plethysmograph recordings showing an example of FRC loss between inflations in an Am pup. FRC loss between inflations measured in (b) pups treated with saline ( $S$, filled circles) or amiloride (Am, open circles) and (c) pups administered adrenaline and then treated with saline ( $\mathrm{S} / \mathrm{Ad}$, open triangles) or amiloride ( $\mathrm{Am} / \mathrm{Ad}$, filled triangles). FRC loss was significantly greater in Am as compared with $S$ pups $\left({ }^{*} P<0.05\right)$. The dashed line represents FRC loss from the $S$ pups in $\mathbf{a}$. The dotted line indicates zero change in the rate of FRC loss. Am, treated with amiloride; Am/ $\mathrm{Ad}$, treated with amiloride and adrenaline; FRC, functional residual capacity; $\mathrm{S}$, treated with saline; $S / A d$, treated with saline and adrenaline.

$(P<0.05)$ but did not change in Am pups $(P>0.05$; Figure $2 \mathbf{b})$. At inflation 40, FRC loss was reduced in $S$ pups as compared with Am pups $(-3.5 \pm 0.6$ vs. $-5.2 \pm 0.6 \mathrm{ml} / \mathrm{kg} / \mathrm{s}$, respectively; $P<0.05)$. By contrast, FRC loss in S/Ad and Am/Ad pups was less than that observed in $\mathrm{S}$ and Am pups. In S/Ad and $\mathrm{Am} / \mathrm{Ad}$ pups, FRC loss was $-2.0 \pm 0.4$ and $-2.0 \pm 0.4 \mathrm{ml} / \mathrm{kg} / \mathrm{s}$, respectively, at inflation 20 and $-1.0 \pm 0.8$ and $-1.5 \pm 0.3 \mathrm{ml} / \mathrm{kg} / \mathrm{s}$, respectively, at inflation 120 (Figure 2c).

Variation in lung aeration at FRC. The coefficient of variation (CV) in lung aeration at FRC was similar in S and Am pups, although it tended to be higher (more varied aeration) in Am 

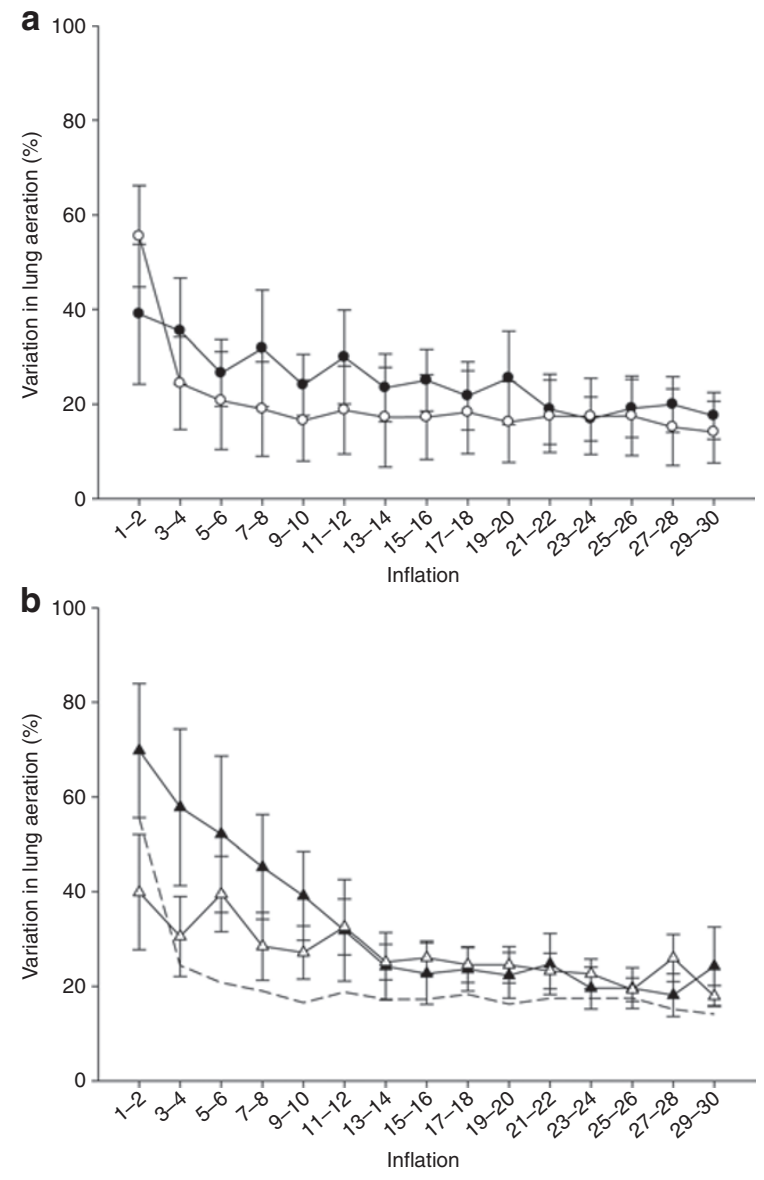

Figure 3. Variation in lung aeration at FRC measured in (a) pups administered saline ( $\mathrm{S}$, open circles) or amiloride (Am, filled circles) and (b) pups administered adrenaline and then treated with saline (S/Ad, open triangles) or amiloride (Am/Ad, filled triangles). The dashed line represents the variation in lung aeration at FRC from the S-treated pups. FRC, functional residual capacity.

pups (Figure 3a). In S pups, the uniformity of lung aeration increased (lower CV) from inflations $1-2$ to inflations 3-4 $(55.5 \pm 10.7 \%$ vs. $24.4 \pm 9.8 \%$, respectively; $P<0.05)$ and then remained $<20 \%$ for the next 30 inflations. In Am pups, the variation in lung aeration tended to be greater than that in $S$ pups and remained $>20 \%$ for the first 20 inflations (Figure 3a). The variation in lung aeration decreased (increased uniformity) with increasing inflation number in Am/Ad pups (69.8 $\pm 14.2 \%$ at inflations $1-2$ vs. $39.9 \pm 12.2 \%$ at inflations $11-12$; $P$ $<0.001$ ) (Figure 3b) and tended to be elevated compared with S/Ad pups for the first 12 inflations.

Peak inspiratory pressure. Although collectively, peak inspiratory pressure (PIP) was not significantly different between S and Am pups over the first 121 inflations, it tended to be lower in $S$ as compared with Am pups (Figure 4a). However, the rate of decrease in PIP was significantly $(P<0.001)$ greater in $S$ as compared with Am pups, between inflations 5 and 40. Over this time, PIP decreased from $33.7 \pm 0.2$ to $23.3 \pm 0.8 \mathrm{cmH}_{2} \mathrm{O}$ in $\mathrm{S}$ pups $(P<0.05)$ and from $32.1 \pm 0.4$ to $27.1 \pm 1.8 \mathrm{cmH}_{2} \mathrm{O}$ in Am pups $(P<0.05)$. PIPs were similar in S/Ad and $\mathrm{Am} / \mathrm{Ad}$
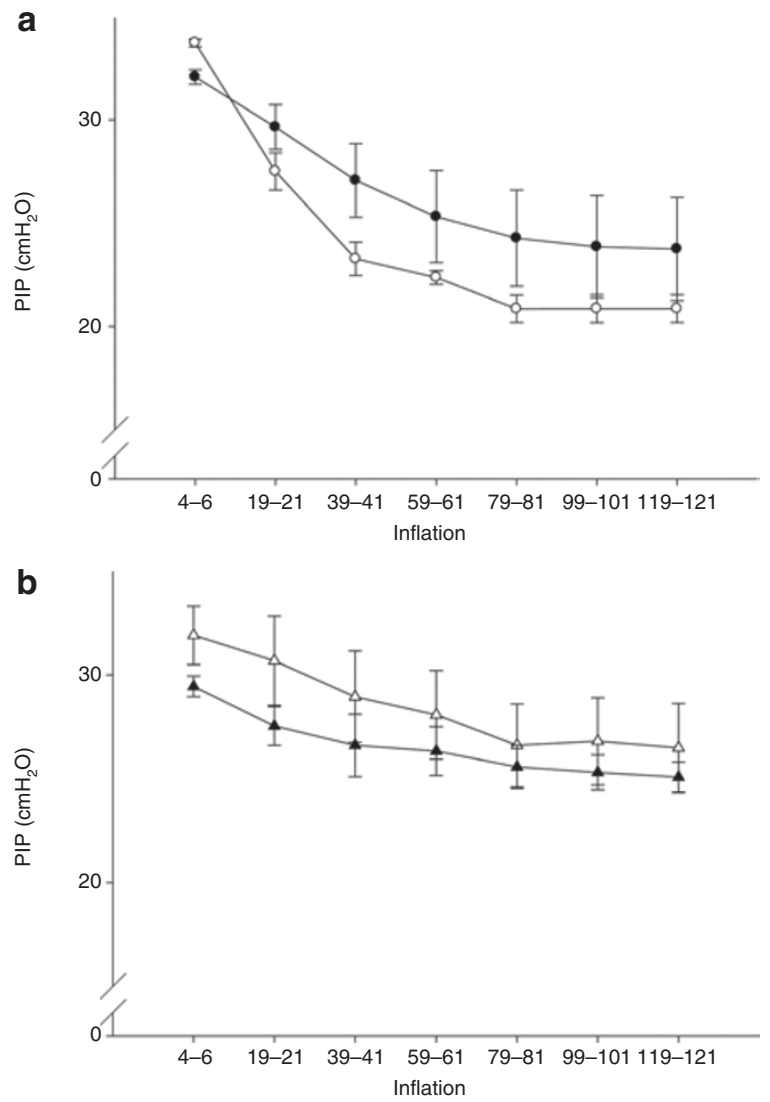

Figure 4. PIP measured in (a) pups treated with saline (S, open circles) or amiloride (Am, filled circles) and (b) pups administered adrenaline and then treated with saline (S/Ad, open triangles) or amiloride (Am/Ad, filled triangles). The rate at which PIP decreased between inflations 5-40 was significantly greater in S-treated than Am-treated pups $(P<0.001)$. PIP, peak inspiratory pressure.

pups and gradually decreased from $\sim 31 \mathrm{cmH}_{2} \mathrm{O}$ at inflation 5 to $\sim 27 \mathrm{cmH}_{2} \mathrm{O}$ at inflation 120 (Figure $4 \mathrm{~b}$ ).

Compliance. Lung compliance was greater in pups that did not receive adrenaline $(\mathrm{S}$ and $\mathrm{Am})$ than those that did receive adrenaline (S/Ad and Am/Ad) $(P<0.05)$. Within the groups of pups that did or did not receive adrenaline, there were no differences between those that received saline and those that received amiloride $(P>0.05)$.

\section{Study B}

The results of Study B can be found in the Supplementary Data online.

\section{DISCUSSION}

In ventilated near-term newborn rabbit pups, amiloride (an $\mathrm{ENaC}$ inhibitor) and adrenaline had little effect on lung aeration and FRC development at birth (Figure 1). Indeed, it was difficult to observe any differences in lung aeration between experimental groups in PC X-ray videos (Supplementary Videos S1-S4 online). Because lung aeration was closely associated with airway liquid clearance (Supplementary Data online), our results imply that ventilation played a more prominent role 


\section{Articles | Siewetal.}

in airway liquid clearance at birth than did ENaCs. Pups given amiloride did, however, require higher PIPs to achieve the target tidal volume, which most probably indicates that the lungs contained more liquid within the airways and were less efficient at clearing this volume. This is consistent with the finding that Am pups had a greater FRC loss between inflations. Therefore, $\mathrm{ENaC}$ activity may help to prevent liquid re-entry into the airspaces when transpulmonary hydrostatic pressure gradients are low (i.e., at positive end expiratory pressure (PEEP)) and higher inflation pressures are required in the absence of $\mathrm{ENaC}$ activity.

Adrenaline-induced activation of $\mathrm{ENaCs}$ is hypothesized as the primary mechanism responsible for airway liquid clearance in the newborn $(1,7,8,11,12,19-22)$. However, PC X-ray imaging has recently revealed that lung aeration is predominantly $(\sim 90 \%)$ associated with inspiratory efforts and can occur rapidly (within three to five breaths during 10-15 s) in spontaneously breathing newborns delivered by cesarean section $(17,18)$. Indeed, lung aeration did not occur in the absence of breathing activity but did occur in response to ventilation many hours after death, when $\mathrm{ENaC}$ activity was expected to be low $(17,18)$. Although transpulmonary hydrostatic pressures were not directly measured, these data provide compelling evidence that hydrostatic pressures generated during inspiration play a major role in airway liquid clearance at birth; that is, airway pressures increase above interstitial tissue pressures and displace liquid across the semipermeable pulmonary epithelium from the airways into the interstitial tissue (18). This can be accomplished during both artificial ventilation and spontaneous breathing. In the former, an artificial inflation increases airway pressure above interstitial tissue pressure to promote liquid clearance from the airways. In the latter, the increase in thoracic volume resulting from inspiration decreases interstitial tissue pressure to a greater extent than the decrease in airway pressure, thereby generating the pressure gradient that promotes airway liquid clearance. We have previously calculated the flux rates for liquid movement across the epithelium during lung aeration (17), and they are orders of magnitude greater than the maximal reabsorption rates that can be induced with adrenaline $(21,23,24)$. Because gas exchange must be initiated shortly after birth, transpulmonary hydrostatic pressure gradients are a likely mechanism that can remove airway liquid at a rapid rate to meet this need.

We consider that the dose of amiloride used in this study to achieve a final concentration of $10^{-4} \mathrm{~mol} / \mathrm{l}$ in airway liquid was sufficient to suppress ENaC activity in this model. This dose induces respiratory distress in spontaneously breathing neonatal rabbits (8), causes microscopic differences in liquid clearance (25), and blocks adrenaline- and vasopressin-induced lung liquid reabsorption in fetal sheep $(19,21)$. Although amiloride may not have blocked all $\mathrm{ENaC}$ activity in this study, we are confident that amiloride was effective. Amiloride can change ion transport within $40 \mathrm{~s}$ of reaching the pulmonary epithelium (4), and in our experiment, $\geq 5$ min separated the administration of amiloride from the first physiological measurement. Indeed, we observed two biological responses to amiloride: an increase in FRC loss between inflations and a requirement for higher PIPs.
Although the transpulmonary hydrostatic pressure gradient may be able to clear liquid from the airways during inflation, we have previously shown that FRC tends to decrease between breaths/inflations in both spontaneously breathing and mechanically ventilated term and preterm newborn rabbits $(17,18,26,27)$. This decreasing FRC represents the re-entry of liquid into the airways from the interstitial tissue compartment (18) when transpulmonary hydrostatic pressure gradients are low, as during expiration. Indeed, PC X-ray imaging has shown that the air-liquid interface can retreat proximally back up the airways during expiration in spontaneously breathing newborn rabbits, particularly early in the aeration process, and then move distally again during the subsequent inspiration (18). The need for higher PIPs in Am pups may be due to the absence of a $\mathrm{Na}^{+}$reabsorption-induced osmotic gradient that normally assists in airway liquid clearance $(3,17)$.

The force that promotes liquid re-entry into the air spaces at rest could result from elevated interstitial tissue pressures that normally occur after birth (28). Because liquid is cleared from the airways at a much faster rate than from the interstitial tissue, it accumulates in perivascular cuffs that surround pulmonary vessels $(29,30)$. This transiently $(\sim 4 \mathrm{~h})$ increases interstitial tissue pressures to $\sim 6 \mathrm{cmH}_{2} \mathrm{O}$ (28), and the chest wall expands to accommodate the extra volume caused by air entry and the tissue accumulation of liquid that has been cleared from the airways $(18,31)$. Although excess interstitial tissue liquid is eventually cleared, via the vascular and lymphatic systems, this process can take up to $4 \mathrm{~h}$ (28). Until this liquid is cleared, high interstitial tissue pressures $\left(\sim 6 \mathrm{cmH}_{2} \mathrm{O}\right)$ (28) must provide a hydrostatic pressure gradient for water movement back into the airways when the lung is at rest. Therefore, successful lung liquid clearance requires the clearance of liquid from the airways as well as a mechanism to ensure that displaced liquid does not re-enter the airways but remains within the interstitial tissue to be cleared from the lungs.

Adrenaline-induced activation of $\mathrm{ENaCs}$ at birth generates a large $\mathrm{Na}^{+}$and $\mathrm{Na}^{+}$-linked $\mathrm{Cl}^{-}$flux from the lung lumen into the interstitial tissue compartment, which reverses the osmotic gradient across the pulmonary epithelium and increases liquid reabsorption (21). It is possible that this mechanism generates an osmotic gradient that opposes the elevated interstitial tissue pressure caused by liquid accumulation within the interstitial tissue compartment at end expiration. This is consistent with our findings that pups given amiloride struggled to maintain FRC between inflations, whereas adrenaline reduced FRC loss between inflations (Figure 2a). Similarly, near-term mechanically ventilated newborn rabbit pups given amiloride had smaller perivascular fluid cuffs than those given saline (6).

Previous studies have demonstrated that amiloride inhibits adrenaline-induced lung liquid reabsorption in term fetal sheep (19), whereas in this study amiloride marginally reduced the effect of adrenaline on the rate of FRC loss (Figure 2b). The reasons for this discrepancy are unclear, although the previous studies were performed in a liquid-filled fetal lung in the absence of lung aeration that might activate physiological mechanisms, in addition to ENaCs, that oppose liquid reflux at 
FRC. However, it is also possible that adrenaline-treated pups were less mature, which would be expected to reduce the effect of adrenaline $(23,32)$. The lower lung compliances in adrenaline-treated than non-adrenaline-treated pups suggest that the adrenaline-treated pups may have been more immature (see the Compliance section).

Study B focused on determining if aerated airways were mainly filled with air or if the lung liquid remained in the airways, coexisting with the air, and was cleared more slowly. We found that a thin layer of liquid lined the inner surface of the epithelium and occupied $\sim 2 \%$ of the cross-sectional surface area of these airways. Therefore, liquid and air do not coexist within the distal airways following lung aeration and so lung gas volume at FRC probably reflects the amount of airway liquid clearance. This is consistent with other physiological findings; a thick layer of liquid would greatly impede gas exchange, and lung compliance markedly increases as the lung aerates $(26,27)$. Fluorescein isothiocyanate-conjugated albumin (FITC-albumin) was also observed within the interstitial tissue compartment in some regions of the lungs. This probably reflects ventilation-induced injury to the epithelium that allowed FITC-albumin to penetrate into the interstitial tissue compartment rather than the possibility that FITC-albumin was permeable across the intact pulmonary epithelium (Supplementary Data, Figure S1 online).

The results of Study B confirm that lung aeration correlates with lung liquid clearance and that the two fluids do not significantly coexist in the airways after birth. PC X-ray imaging therefore is a novel method to investigate lung liquid clearance at birth that, unlike more traditional methods of measuring lung liquid clearance $(24,32-35)$, is not complicated by the nonuniform distribution of the impermeable tracer throughout the lungs, the difficulty of sampling fluid from the alveoli (site of liquid secretion/ absorption), and the difficulty of distinguishing between liquid within airspaces from liquid within interstitial tissue.

This study investigated the relative roles of $\mathrm{ENaCs}$ and ventilation in the development and maintenance of FRC in mechanically ventilated near-term newborn rabbit pups. We found that FRC development was not dependent on $\mathrm{ENaC}$ activity and that increased airway pressures can compensate for the absence of $\mathrm{ENaC}$ activity and facilitate liquid clearance. We suggest that transpulmonary hydrostatic pressures are the primary mechanism for airway liquid clearance at birth and that $\mathrm{ENaC}$ activation helps to reduce liquid reflux between inflations when airway pressures are low and interstitial tissue pressures are elevated due to liquid accumulation.

\section{METHODS}

All experiments were approved by SPring- 8 and/or by animal ethics committees of the School of Biomedical Science, Monash University. Study A was performed at the SPring- 8 synchrotron in Japan, whereas Study B was performed at Monash University, Clayton, Australia.

\section{Study A: Effect of ENaC Inhibition and Adrenaline on Lung Aeration After Birth}

Experimental protocol. Pregnant near-term New Zealand white rabbits (30 d gestation; term $=32 \mathrm{~d}$ ) were anesthetized using Rapinovet $(12 \mathrm{mg}$ / kg i.v. bolus, Schering-Plough Animal Health, Millsboro, DE) and intubated. Anesthesia was maintained by isoflurane inhalation $(1.5-4 \%$,
Delvet, Seven Hills, Australia). Pups were partially delivered by cesarean section, sedated (Nembutal, sodium pentobarbitone $0.1 \mathrm{mg}$ i.p., Abbott Laboratories, North Chicago, IL), and randomized within the litter to be intubated with an endotracheal tube (18G) containing $0.1 \mathrm{ml}$ of saline or amiloride $(1.35 \mathrm{mmol} / \mathrm{l}$; amiloride hydrochloride hydrate; Sigma-Aldrich, St Louis, MO). Airway liquid was repeatedly withdrawn and replaced (23) to mix amiloride within the airway liquid to a final concentration of $10^{-4} \mathrm{~mol} / \mathrm{l}$, assuming pups weighed $50 \mathrm{~g}$ (on the basis of previous observations) and airway liquid volumes were $25 \mathrm{ml} / \mathrm{kg}$. Another group of pups received adrenaline $(1.5 \mu \mathrm{g}$ in $0.15 \mathrm{ml}$; SigmaAldrich) via the umbilical vein and were randomized to receive either $0.1 \mathrm{ml}$ saline or $0.1 \mathrm{ml}$ amiloride into the airway liquid as described above. The umbilical cord was then cut and the pup delivered.

The pup was positioned upright in a warm water-filled $\left(40^{\circ} \mathrm{C}\right)$ plethysmograph (head out) within the imaging hutch (custom-built; see refs. 18,26). The endotracheal tube was connected to a custombuilt ventilator (36), and ventilation and image acquisition were commenced. Time from intratracheal drug administration to ventilation was $\geq 5$ min but often longer ( $~ 8 \mathrm{~min})$. Pups were ventilated with a tidal volume of $10 \mathrm{ml} / \mathrm{kg}$; pup weight was estimated at $50 \mathrm{~g}$ in $\mathrm{S}$ and $\mathrm{Am}$ groups but was directly measured in S/Ad and Am/Ad groups. A PEEP of $3 \mathrm{cmH}_{2} \mathrm{O}$ and ventilation rate of 24 inflations per minute was used for $7 \mathrm{~min}$. Changes in lung gas volumes were measured by plethysmography, and airway pressures were recorded digitally (Powerlab; AD Instruments; Sydney, Australia). Following the experiment, pups were killed with an overdose of sodium pentobarbitone ( $>100 \mathrm{mg} / \mathrm{kg}$ i.p.).

PC X-ray imaging. PC X-ray imaging $(14,15,18)$ was performed at an $\mathrm{X}$-ray energy of $24 \mathrm{keV}$. Pups were located $2 \mathrm{~m}$ upstream of the fiber optics camera (Hamamatsu C9300-124F; Hamamatsu, Hamamatsu City, Japan) with an effective pixel size of $16.2 \mu \mathrm{m}$ and an active field of view of 32 (horizontal) $\times 32$ (vertical) $\mathrm{mm}^{2}$. Inflation onset triggered the camera to acquire a train of seven images, $300 \mathrm{~ms}$ apart; the first three images were acquired during inspiration, and the last one or two were at FRC. PC X-ray videos show approximately the first 10 inflations and are played at 5 images per second. Supplementary Video S1 online is of an S pup; Supplementary Video S2 online is of an Am pup; Supplementary Video S3 online is of an S/Ad pup; and Supplementary Video S4 online is of an Am/Ad pup.

Plethysmograph analysis. FRC, FRC loss between inflations, PIP, and lung compliance were measured. FRC was the average lung gas volume during expiration. FRC loss was the amount of gas volume lost during expiration, when airway pressures were at PEEP; this is determined as the decrease in lung gas volume between the inflection point when the lungs had initially deflated to PEEP and just before onset of the next inflation (Figure 2a). PIP was the pressure used to inflate the lungs. Compliance was equal to the tidal volume divided by PIP minus PEEP. Results are presented as the average of three inflations and measured at inflations $5 \pm 1$ (i.e., average of inflations 4-6), inflations $20 \pm 1$, and at every $20 \pm 1$ inflation for 120 inflations.

Image analysis. PC X-ray images of the lungs at FRC were analyzed to assess the uniformity of lung aeration using a phase retrieval analysis that calculates lung gas volumes from the images $(27,37,38)$. PC X-ray images were divided into four quadrants, vertically down the spine and horizontally at the seventh rib. Phase retrieval algorithms were applied to each quadrant to determine the lung gas volume. This was normalized to the maximum volume achieved in that quadrant to account for the differences in lung size within each quadrant. The uniformity of lung aeration was assessed at each time point using the four normalized gas volume values, and it was used to calculate a CV between all four quadrants of the PC X-ray image (27). Higher CVs indicated more heterogeneous aeration, whereas lower CVs indicated more uniform aeration. Results are presented as an average of two inflations from the start of ventilation for a total of 30 inflations.

Statistical analysis. Results are presented as mean \pm SEM, and all data were checked for normality and tested for equal variance. Changes in FRC, FRC loss, PIP, and CV were analyzed using a two-way repeated-measures ANOVA and a least significant difference post hoc test. 
Differences in the rate of decrease in PIP between Am and S were analyzed by statistically comparing the slopes of the two lines. A $P$ value of $<0.05$ indicated a significant difference.

\section{Study B: Relationship Between Lung Aeration and Airway Liquid Removal}

Experimental protocol. Preterm New Zealand white rabbit pups ( $28 \mathrm{~d}$ of gestational age) were delivered and intubated as described for Study A. Endotracheal tubes were preloaded with $0.1 \mathrm{ml}$ of FITC-albumin $(400$ $\mu \mathrm{g} / \mathrm{ml}$; Sigma-Aldrich) and injected into the lung liquid. Because albumin does not cross the pulmonary epithelium (39), florescence within the airway lumen reflects the presence of liquid within the airway. FITCalbumin-labeled lung liquid was slowly and repeatedly withdrawn and reinjected into the lung $\sim 20$ times. The umbilical cord was then cut, and the pup was placed in a warm $\left(40^{\circ} \mathrm{C}\right)$ water-filled plethysmograph (head out) and connected to a mechanical ventilator (36). Pups were ventilated with a tidal volume of $7 \mathrm{ml} / \mathrm{kg}$ and a PEEP of $5 \mathrm{cmH}_{2} \mathrm{O}$ at 24 inflations per minute for $7 \mathrm{~min}$ and then killed with sodium pentobarbitone $(>100 \mathrm{mg} /$ $\mathrm{kg}$ i.p.). The lungs were removed, re-inflated to PIP, and then deflated to $20 \mathrm{cmH}_{2} \mathrm{O}$ before they were snap frozen in dry ice-chilled isopentane (Sigma-Aldrich). Controls were fetal rabbits that had FITC-albumin mixed with lung liquid and were killed without being ventilated.

Histology. Lungs were sectioned at $16 \mu \mathrm{m}$ in a cryostat, and approximately every 35 th section was collected. Fluorescent images of distal air sacs lined with FITC-albumin were acquired at a magnification of 20x, and image location was determined from a MD3 Microscope Digitizer (AccuStage, Shoreview, MN). Sections were then fixed in acetone, stained with hematoxylin, and light microscope images were acquired at the same coordinates to merge the two images (ImagePro Plus; Media Cybernetics, Rockville, MD). In ventilated lungs, only aerated airspaces within the image were analyzed. In both ventilated and nonventilated lungs, the area of FITC-albumin within the airspace was expressed as a percentage of the total cross-sectional area of the airspace for those regions.

Statistical analysis. Results are presented as means \pm SEM, and all data were checked for normality and equal variance before analysis. The difference between the ventilated and unventilated groups was analyzed using a Student's $t$ test. A $P$ value of $<0.05$ indicated a significant difference.

\section{SUPPLEMENTARY MATERIAL}

Supplementary material is linked to the online version of the paper at http://www.nature.com/pr

\section{STATEMENT OF FINANCIAL SUPPORT}

This research was supported by the Australian Research Council, the Australian National Health and Medical Research Council (NHMRC), and the Victorian Government's Operational Infrastructure Support Program. We acknowledge travel funding provided by the International Synchrotron Access Program (ISAP) managed by the Australian Synchrotron and funded by the Australian government. We also gratefully acknowledge the support provided by the SPring-8 synchrotron facility (Japan) granted by the SPring-8 Program Review Committee (proposal nos. 2008A0002/2009A0002). M.L.S. was the recipient of an Australian NHMRC postgraduate scholarship. M.J.K. was the recipient of an ARC postdoctoral fellowship. A.B.t.P. is a recipient of a Veni-grant from The Netherlands Organisation for Health Research and Development (ZonMw), part of the Innovational Research Incentives Scheme Veni-Vidi-Vici (project no. 91612027).

\section{REFERENCES}

1. Hooper SB, Harding R. Role of aeration in the physiological adaptation of the lung to air-breathing at birth. Curr Respir Med Rev 2005;1:185-95.

2. te Pas AB, Davis PG, Hooper SB, Morley CJ. From liquid to air: breathing after birth. J Pediatr 2008;152:607-11.

3. Hooper SB, te Pas AB, Lewis RA, Morley CJ. Establishing functional residual capacity at birth. NeoReviews 2010;11:474-83.

4. Collett A, Ramminger SJ, Olver RE, Wilson SM. Beta-adrenoceptormediated control of apical membrane conductive properties in fetal distal lung epithelia. Am J Physiol Lung Cell Mol Physiol 2002;282:L621-30.
5. Rafii B, Gillie DJ, Sulowski C, et al. Pulmonary oedema fluid induces nonalpha-ENaC-dependent $\mathrm{Na}(+)$ transport and fluid absorption in the distal lung. J Physiol (Lond) 2002;544(Pt 2):537-48.

6. Song GW, Sun B, Curstedt T, Grossmann G, Robertson B. Effect of amiloride and surfactant on lung liquid clearance in newborn rabbits. Respir Physiol 1992;88:233-46.

7. Hummler E, Barker P, Gatzy J, et al. Early death due to defective neonatal lung liquid clearance in alpha-ENaC-deficient mice. Nat Genet 1996;12:325-8.

8. O’Brodovich H, Hannam V, Seear M, Mullen JB. Amiloride impairs lung water clearance in newborn guinea pigs. J Appl Physiol 1990;68:1758-62.

9. Helve O, Andersson S, Kirjavainen T, Pitkänen OM. Improvement of lung compliance during postnatal adaptation correlates with airway sodium transport. Am J Respir Crit Care Med 2006;173:448-52.

10. Helve O, Pitkänen OM, Andersson S, O'Brodovich H, Kirjavainen T, Otulakowski G. Low expression of human epithelial sodium channel in airway epithelium of preterm infants with respiratory distress. Pediatrics 2004;113:1267-72.

11. Hooper SB, Harding R. Fetal lung liquid: a major determinant of the growth and functional development of the fetal lung. Clin Exp Pharmacol Physiol 1995;22:235-47.

12. Olver RE, Walters DV, M Wilson S. Developmental regulation of lung liquid transport. Annu Rev Physiol 2004;66:77-101.

13. Strang LB. Fetal lung liquid: secretion and reabsorption. Physiol Rev 1991;71:991-1016.

14. Kitchen MJ, Paganin D, Lewis RA, Yagi N, Uesugi K, Mudie ST. On the origin of speckle in $\mathrm{x}$-ray phase contrast images of lung tissue. Phys Med Biol 2004;49:4335-48.

15. Lewis RA, Yagi N, Kitchen MJ, et al. Dynamic imaging of the lungs using X-ray phase contrast. Phys Med Biol 2005;50:5031-40.

16. YagiN, Suzuki Y,Umetani K, Kohmura Y, Yamasaki K. Refraction-enhanced $\mathrm{x}$-ray imaging of mouse lung using synchrotron radiation source. Med Phys 1999;26:2190-3.

17. Siew ML, Wallace MJ, Kitchen MJ, et al. Inspiration regulates the rate and temporal pattern of lung liquid clearance and lung aeration at birth. J Appl Physiol 2009;106:1888-95.

18. Hooper SB, Kitchen MJ, Wallace MJ, et al. Imaging lung aeration and lung liquid clearance at birth. FASEB J 2007;21:3329-37.

19. Hooper SB, Wallace MJ, Harding R. Amiloride blocks the inhibition of fetal lung liquid secretion caused by AVP but not by asphyxia. J Appl Physiol 1993;74:111-5.

20. Wallace MJ, Hooper SB, Harding R. Effects of elevated fetal cortisol concentrations on the volume, secretion, and reabsorption of lung liquid. Am J Physiol 1995;269(4 Pt 2):R881-7.

21. Olver RE, Ramsden CA, Strang LB, Walters DV. The role of amilorideblockable sodium transport in adrenaline-induced lung liquid reabsorption in the fetal lamb. J Physiol (Lond) 1986;376:321-40.

22. Jain L, Eaton DC. Physiology of fetal lung fluid clearance and the effect of labor. Semin Perinatol 2006;30:34-43.

23. Hooper SB, Harding R. Effect of beta-adrenergic blockade on lung liquid secretion during fetal asphyxia. Am J Physiol 1989;257(4 Pt 2):R705-10.

24. Walters DV, Olver RE. The role of catecholamines in lung liquid absorption at birth. Pediatr Res 1978;12:239-42.

25. Ramsden CA, Markiewicz M, Walters DV, et al. Liquid flow across the epithelium of the artificially perfused lung of fetal and postnatal sheep. J Physiol (Lond) 1992;448:579-97.

26. Siew ML, Te Pas AB, Wallace MJ, et al. Positive end-expiratory pressure enhances development of a functional residual capacity in preterm rabbits ventilated from birth. J Appl Physiol 2009;106:1487-93.

27. te Pas AB, Siew M, Wallace MJ, et al. Establishing functional residual capacity at birth: the effect of sustained inflation and positive end-expiratory pressure in a preterm rabbit model. Pediatr Res 2009;65:537-41.

28. Miserocchi G, Poskurica BH, Del Fabbro M. Pulmonary interstitial pressure in anesthetized paralyzed newborn rabbits. J Appl Physiol 1994;77:2260-8.

29. Bland RD, McMillan DD, Bressack MA, Dong L. Clearance of liquid from lungs of newborn rabbits. J Appl Physiol 1980;49:171-7. 
30. Raj JU, Bland RD. Lung luminal liquid clearance in newborn lambs. Effect of pulmonary microvascular pressure elevation. Am Rev Respir Dis 1986;134:305-10.

31. Enhorning G, Robertson B, Milne E, Wagner R. Radiologic evaluation of the premature newborn rabbit after pharyngeal deposition of surfactant. Am J Obstet Gynecol 1975;121:475-80.

32. Brown MJ, Olver RE, Ramsden CA, Strang LB, Walters DV. Effects of adrenaline and of spontaneous labour on the secretion and absorption of lung liquid in the fetal lamb. J Physiol (Lond) 1983;344:137-52.

33. Olver RE, Schneeberger EE, Walters DV. Epithelial solute permeability, ion transport and tight junction morphology in the developing lung of the fetal lamb. J Physiol (Lond) 1981;315:395-412.

34. Bland RD, Bressack MA, McMillan DD. Labor decreases the lung water content of newborn rabbits. Am J Obstet Gynecol 1979;135:364-7.
35. Wallace MJ, Hooper SB, Harding R. Regulation of lung liquid secretion by arginine vasopressin in fetal sheep. Am J Physiol 1990;258(1 Pt 2):R104-11.

36. Kitchen MJ, Habib A, Fouras A, et al. A new design for high stability pressure-controlled ventilation for small animal lung imaging. J Instrum Tech 2010;5:T02002.

37. Kitchen MJ, Lewis RA, Morgan MJ, et al. Dynamic measures of regional lung air volume using phase contrast $\mathrm{x}$-ray imaging. Phys Med Biol 2008;53:6065-77.

38. Siew ML, Te Pas AB, Wallace MJ, et al. Surfactant increases the uniformity of lung aeration at birth in ventilated preterm rabbits. Pediatr Res 2011;70:50-5.

39. Egan EA, Olver RE, Strang LB. Changes in non-electrolyte permeability of alveoli and the absorption of lung liquid at the start of breathing in the lamb. J Physiol (Lond) 1975;244:161-79. 